\title{
Superimposed Training based Estimation of Sparse MIMO Channels for Emerging Wireless Networks
}

\author{
${ }^{\S}$ Babar Mansoor, ${ }^{\S}$ Syed Junaid Nawaz, ${ }^{*}$ Bilal Amin, ${ }^{\dagger}$ Shree K. Sharma, and ${ }^{\ddagger}$ Mohmammad N. Patwary \\ $\S$ Department of Electrical Engineering, COMSATS Institute of Information Technology, Islamabad, Pakistan. \\ * Department of Electrical Engineering, COMSATS Institute of Information Technology, Lahore, Pakistan. \\ $\dagger$ SnT - securityandtrust.lu, University of Luxembourg, Luxembourg \\ $\ddagger$ Faculty of Computing Engineering and Sciences, Staffordshire University, Stoke-on-trent, UK. \\ Email: babar_mansoor@comsats.edu.pk, junaidnawaz@ieee.org, bilalamin@ciitlahore.edu.pk, \\ shree.sharma@uni.lu, and m.n.patwary@staffs.ac.uk.
}

\begin{abstract}
Multiple-input multiple-output (MIMO) systems constitute an important part of todays wireless communication standards and these systems are expected to take a fundamental role in both the access and backhaul sides of the emerging wireless cellular networks. Recently, reported measurement campaigns have established that various outdoor radio propagation environments exhibit sparsely structured channel impulse response (CIR). We propose a novel superimposed training (SiT) based up-link channels' estimation technique for multipath sparse MIMO communication channels using a matching pursuit (MP) algorithm; the proposed technique is herein named as superimposed matching pursuit (SI-MP). Subsequently, we evaluate the performance of the proposed technique in terms of mean-square error (MSE) and bit-error-rate (BER), and provide its comparison with that of the notable first order statistics based superimposed least squares (SI-LS) estimation. It is established that the proposed SI-MP provides an improvement of about $2 \mathrm{~dB}$ in the MSE at signal-to-noise ratio (SNR) of $12 \mathrm{~dB}$ as compared to SI-LS, for channel sparsity level of $21.5 \%$. For BER $=10^{-2}$, the proposed SI-MP compared to SI-LS offers a gain of about $3 \mathrm{~dB}$ in the SNR. Moreover, our results demonstrate that an increase in the channel sparsity further enhances the performance gain.
\end{abstract}

Keywords: MIMO, sparse, superimposed training, firstorder statistics, compressed sensing, matching pursuit, channel estimation

\section{INTRODUCTION}

The emerging fifth generation $(5 \mathrm{G})$ cellular networks are generally envisioned to deliver a decrease in the latency in the order of tens, an increase in energy efficiency in the order of hundreds, and an increase in system capacity in the order of thousands [1,2]. To attain these targets, the emerging 5G cellular networks are thought to adopt multitier cellular architectures with macrocells, dense small sized cells, relays, and device-to-device (D2D) communication links. A large amount of antennas employed at the cellular base stations (BSs) has the potential to offer numerous advantages in terms of high data rates, improved spectral efficiency,

The first three authors would like to acknowledge the partial financial support by EU ATOM-690750 research project approved under the call H2020-MSCA-RISE-2015. The fourth author would like to acknowledge the partial financial support by the National Research Fund, Luxembourg under the CORE project "SeMiGod". support for a large number of users, low transmit power, high order diversity, improved range of coverage, and better interference suppression. Therefore, such massive multipleinput multiple-output (MIMO) systems have recently received significant attention as a potential candidate for providing promising solutions for both wireless access and backhaul. Further, a large amount of under-utilized millimeter wave (mmWave) spectrum is being investigated for massive MIMO systems to provide the requisite band in order to address high capacity demands of wireless backhaul [2] and wireless cellular network [3].

Various empirical results have established that mmWave massive MIMO channels exhibit spatially sparse structure [4], where only a few dominant paths exist (typically, $3 \sim 5$ multipath). Moreover, in various outdoor radio propagation environments, the impulse response of the channel is observed as sparse $[5,6]$. For example, the channel impulse response vector exhibits sparse structure in a typical cellular communication environment with only a few dominant distant scatterers [6], aeronautical communication channels [7], wideband high frequency communication channels [8], and underwater acoustic communication channels [9], etc. Such sparse propagation channels have only a few dominant multipath components that are largely separated in the delay domain, which makes the estimation of channel a challenging task [10]. However, a prior knowledge about the channel sparsity can assist in obtaining more accurate channel estimates.

In the literature, there exist numerous channel estimation techniques that can be broadly categorized into blind [1113], semi-blind [14-16], and training sequence based [17-19]. Depending upon the channel's time or frequency selectiveness, the training and information sequences can be multiplexed in time or/and frequency domain. Consequently, superimposed training (SiT) sequence based methods have recently gained popularity for the purpose of channel impulse response (CIR) estimation [6,20-22]. In SiT based techniques, a known pilot sequence is superimposed (arithmetically added) over the data sequence, thus it avoids the overhead on data rate by preventing any use of dedicated time/frequency slots for 
the training sequence [22]. These techniques are not only spectrally efficient but also can effectively track the channel variations to combat any sudden blockages.

Over the past few years, compressed sensing (CS) theory [23-25], that exploits the sparse nature of the signals, has gained significant attention. Furthermore, CS has been successfully employed to estimate the CIR in wireless communications - see e.g. [26-28]. In [29], authors have proposed an SiT based channel estimation technique based on the first order statistics of the data for the case of single-input multiple-output (SIMO) time-invariant channels. In [6], an SiT technique that uses compressed sensing and first order statistics of the data, has been proposed for the estimation of sparse multipath channels for single-input single-output (SISO) systems. Among several available reconstruction algorithms used in compressed sensing, the matching pursuit (MP) algorithm exploits the available knowledge of sparse structure of the channel and provides a reasonably good estimate with less computational complexity [5]. Moreover, an SiT based technique for underwater acoustic orthogonal frequency division multiplexing (OFDM) communication systems using the MP algorithm, has been proposed in [10], to obtain the estimate of sparse CIR with a reduced peak-to-average ratio of OFDM channels.

This paper propose a novel SiT based technique for estimation of frequency selective sparse MIMO communication channels. A superimposed training based matching pursuit (SIMP) algorithm for the estimation of such sparse multipath MIMO channels is proposed. In order to demonstrate the usefulness of the proposed technique, a detailed analysis based on bit-error-rate (BER) and mean-square-error (MSE) performance of the system is presented. Moreover, an MSE and BER based comparison of the proposed technique with the superimposed least square (SI-LS) technique devised in [22] is presented.

The rest of this paper is organised as follows: the considered communication system model is presented in Sec. II. The proposed SI-MP technique for sparse MIMO channel estimation is presented in Sec. III. Details of the equalizer used in the simulations is presented in Sec. IV. Simulation results along with a thorough performance analysis is presented in Sec. V. Finally, Sec. VI presents the conclusion.

Notations: Boldface upper case letters, e.g., $\mathbf{H}$ represent matrices. Boldface small case letters represent vectors, e.g., h. Scalar quantities are denoted with small case italic letters, e.g., $h$. Hermitian transpose of a vector is represented as [.] $]^{*}$.

\section{Multiuser Mimo Communication System Model}

The block diagram of the considered multiuser MIMO communication system model is shown in Fig. 1. Let $N$ and $M$ be the number of user terminals (transmitters) and receive antenna array elements, respectively. The signal transmitted from the users propagates through sparse multipath MIMO communication channels with $Q$ and $L$ being the non-zero and the total number of resolvable multipath taps, respectively. Channel estimator (CE) is implemented using the first-order statistics based channel estimation technique in [22] and the proposed

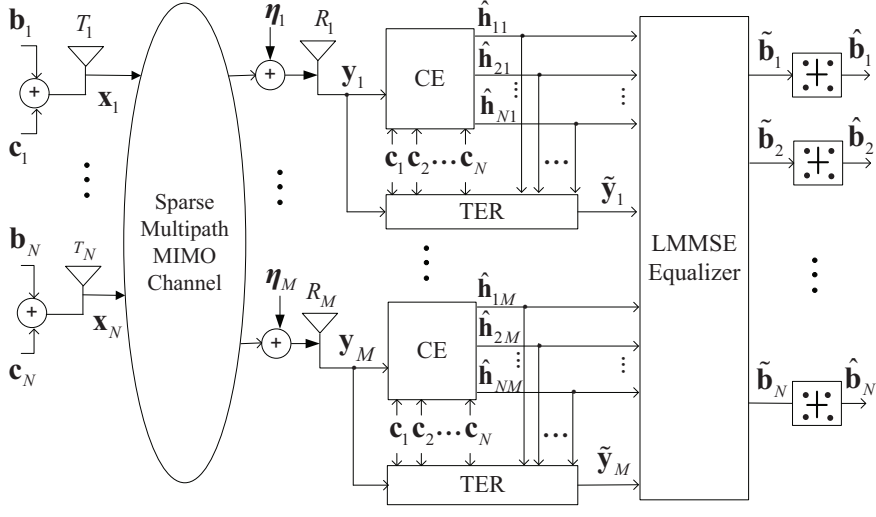

Fig. 1: Block diagram of the considered multiuser MIMO communication system.

superimposed training sequence based matching pursuit (SIMP), detailed later in Section III. Subsequently, trainingsequence effect remover (TER) eliminates the contribution of training sequence after estimation of the CIR and feeds the equalizer with a regularized version of the received signal. Next, a linear minimum mean square equalizer (LMMSE) is implemented to estimate the transmitted information sequence.

Let $\mathbf{b}_{n}=\left[b_{n}(0), b_{n}(1), \cdots, b_{n}(K-1)\right]^{*}$ represent a zeromean information sequence such that $\mathbf{b}_{n}$ is mutually independent for each of the $n^{\text {th }}$ user. A known deterministic and periodic training sequence $\mathbf{c}_{n}=\left[c_{n}(0), c_{n}(1), \cdots, c_{n}(K-1)\right]^{*}$, having a period $P$ such that $c_{n}(k)=c_{n}(k+a P)$ for all integers $k$ and $a$, is superimposed (added) over the information sequence $\mathbf{b}_{\mathbf{n}}$. The superimposed information and training sequences for $n^{\text {th }}$ user terminal is given by,

$$
\mathbf{x}_{n}=\mathbf{b}_{n}+\mathbf{c}_{n} \text {. }
$$

The sequence $\mathbf{x}_{n}=\left[x_{n}(0), x_{n}(1), \cdots, x_{n}(K-1)\right]^{*}$ is transmitted over the sparse MIMO channel such that the impulse response between $n^{\text {th }}$ transmitter and $m^{\text {th }}$ receiver is given by $\mathbf{h}_{n m}=\left[h_{n m}^{0}, h_{n m}^{1}, \cdots, h_{n m}^{L-1}\right]^{*}$. The signal received at time instant $k$ by the $m^{t h}$ antenna element of receive array is given by the following equation,

$$
y_{m}(k)=\sum_{n=1}^{N} \sum_{l=0}^{L-1} h_{n m}^{l} x_{n}(k-l)+\eta_{m}(k),
$$

where $\eta_{m}(k)$ denotes the $k^{t h}$ sample of zero mean additive white Gaussian noise (AWGN) with variance $\sigma_{\eta}^{2}$. The signal received by all the antenna elements of the receive array at time instant $k$ is $\mathbf{y}(k)=\left[y_{1}(k), y_{2}(k), \cdots, y_{M}(k)\right]^{*}$ and is given by,

$$
\mathbf{y}(k)=\sum_{l=0}^{L-1} \mathbf{H}^{l} \mathbf{x}(k-l)+\boldsymbol{\eta}(k),
$$

where $\mathbf{x}(k-l)=\left[x_{1}(k-l), x_{2}(k-l), \cdots, x_{N}(k-l)\right]^{*}$, $\boldsymbol{\eta}(k)=\left[\eta_{1}(k), \eta_{2}(k), \cdots, \eta_{M}(k)\right]^{*}$, and $\boldsymbol{H}^{l}$ represents $M \times$ $N$ channel matrix for a specific delay tap $l$. The temporal sampling yields the following representation of the received signal,

$$
\mathbf{s}(k)=\left[\mathbf{y}^{*}(k+L-1), \mathbf{y}^{*}(k+L-2), \cdots, \mathbf{y}^{*}(k)\right]^{*},
$$




$$
\mathbf{s}(k)=\mathbf{H v}(k)+\breve{\boldsymbol{\eta}}(k),
$$

where the vectors $\mathbf{v}(k)$ and $\breve{\boldsymbol{\eta}}(k)$ are given by,

$$
\begin{aligned}
& \mathbf{v}(k)=\left[\mathbf{x}^{*}(k+L-1), \mathbf{x}^{*}(k+L-2), \cdots, \mathbf{x}^{*}(k)\right]^{*}, \\
& \breve{\boldsymbol{\eta}}(k)=\left[\boldsymbol{\eta}^{*}(k+L-1), \boldsymbol{\eta}^{*}(k+L-2), \cdots, \boldsymbol{\eta}^{*}(k)\right]^{*} .
\end{aligned}
$$

\section{Proposed Sit Based MiMO Channel Estimation}

For the sake of completeness, a brief review of first order statistics of MIMO channels and a notable least square channel estimation method are presented in Sec. III-A and III-B, respectively. The proposed SI-MP channel estimation method is presented in Sec. III-C.

\section{A. First-Order Statistics}

Each user (transmitter) is assigned with a distinct cycle frequency. Suppose for a specific user $n$, the training sequence $c_{n}(k)$ is periodic. The period of the training sequence is $P=\tilde{P} N$, where $\tilde{P} \in \mathbb{Z}^{+}$. The training sequence $c_{n}(k)$ is given as,

$$
c_{n}(k)=\sum_{i=0}^{P-1} c_{i, n} \mathrm{e}^{j(2 \pi i / P) k}, \quad \forall k,
$$

where $j=\sqrt{-1}$ and

$$
c_{i, n}=\frac{1}{P} \sum_{k=0}^{P-1} c_{n}(k) \mathrm{e}^{-j(2 \pi i / P) k}
$$

In (8), $c_{n}(k)$ is chosen such that only $\tilde{P}$ coefficients out of total $P$ are non zero, therefore, $c_{n}(k)$ can be calculated as,

$$
c_{n}(k)=\sum_{i=0}^{\tilde{P}-1} c_{i, n}^{\prime} \mathrm{e}^{j \alpha_{i, n} k}, \quad \forall k,
$$

where $\alpha_{i, n}=2 \pi(i N+n-1) / P$. To compute $c_{n}(k)$, we need a periodic base sequence $\bar{c}_{o}(k)$ that has a period of $\tilde{P}$, [22], in such a way that,

$$
\bar{c}_{i, o}=\frac{1}{\tilde{P}} \sum_{k=0}^{\tilde{P}-1} \bar{c}_{o}(k) \mathrm{e}^{-j(2 \pi i / \tilde{P}) k} .
$$

In order to define the training sequence $\overline{c_{1}}(n)$ having a period $P, \bar{c}_{o}(k)$ is repeated $N$ times. The training sequence of a specific transmitter $n$ can thus be defined as follows [22],

$$
c_{n}(k)=\sigma_{c n} \bar{c}_{1}(k) \mathrm{e}^{j(2 \pi / P)(n-1) k} \text { for } n=1,2, \ldots, N .
$$

The expectation of the received signal $y_{m}(k)$ at the receiver's $m^{\text {th }}$ antenna element can be calculated as:

$$
E\left\{y_{m}(k)\right\}=\sum_{n=1}^{N} \sum_{i=0}^{\tilde{P}-1}\left[\sum_{l=0}^{L-1} c_{i, n}^{\prime} h_{n m}^{l} \mathrm{e}^{-j \alpha_{i, n} l}\right] \mathrm{e}^{j \alpha_{i, n} k} .
$$

For $n_{1} \neq n_{2}$, we have $\alpha_{i_{1}, n_{1}} \neq \alpha_{i_{2}, n_{2}}$ for any $i_{1}, i_{2} \in$ $0,1, \ldots, \tilde{P}-1$. Let $\mathbf{d}_{n m}=\left[d_{n m, 0}, d_{n m, 1}, \cdots, d_{n m,(\tilde{P}-1)}\right]^{*}$, where $d_{n m, i}$ is given by,

$$
d_{n m, i}=\sum_{l=0}^{L-1} c_{i, n}^{\prime} h_{n m}^{l} \mathrm{e}^{-j \alpha_{i, n} l}
$$

The mean square consistent estimate $\hat{\mathbf{d}}_{n m}=$ $\left[\hat{d}_{n m, 0}, \hat{d}_{n m, 1}, \cdots, \hat{d}_{n m,(\tilde{P}-1)}\right]^{*}$ of $\mathbf{d}_{n m}$ can be obtained, by computing its coefficient as given below,

$$
\hat{d}_{n m, i}=\frac{1}{T} \sum_{k=0}^{T-1} y_{m}(k) \mathrm{e}^{-j \alpha_{i, n} k},
$$

where $T$ is the number of received symbols, as $T \rightarrow \infty$, the $\hat{d}_{n m, i} \rightarrow d_{n m, i}$. The relationship given in (14) can also be written in a vector form as,

$$
\hat{\mathbf{d}}_{n m}=\mathbf{C}_{n} \mathbf{h}_{n m},
$$

where $\mathbf{C}_{n}$ can be obtained as

$$
\mathbf{C}_{n}=\operatorname{diag}\left\{c_{0, n}^{\prime}, c_{1, n}^{\prime}, \cdots, c_{(\tilde{P}-1), n}^{\prime}\right\} \mathbf{V}_{n},
$$

where $\mathbf{V}_{n}$ can be found as,

$$
\mathbf{V}_{n}=\left[\begin{array}{cccc}
1 & 1 & \cdots & 1 \\
1 & \mathrm{e}^{-j \alpha_{1, n}} & \cdots & \mathrm{e}^{-j \alpha_{1, n} L} \\
1 & \mathrm{e}^{-j \alpha_{2, n}} & \cdots & \mathrm{e}^{-j \alpha_{2, n} L} \\
\vdots & \vdots & \vdots & \vdots \\
1 & \mathrm{e}^{-j \alpha_{(\tilde{P}-1), n}} & \cdots & \mathrm{e}^{-j \alpha_{(\tilde{P}-1), n} L}
\end{array}\right] .
$$

\section{B. Least Squares}

The least square estimate [22] of the channel can thus be obtained from (15) in the following way

$$
\hat{\mathbf{h}}_{\mathbf{n m}}=\left(\mathbf{C}_{n}^{*} \mathbf{C}_{n}\right)^{-1} \mathbf{C}_{n}^{*} \hat{\mathbf{d}}_{n m} .
$$

For non-zero mean noise, the channel estimate can be obtained by setting $\tilde{P} \geq L+1$, and by omitting the first row from $\mathbf{C}_{n}$ and $\hat{d}_{n m, 0}$ from $\hat{\mathbf{d}}_{n m}$.

\section{Proposed SI-MP}

The error in the estimate $\hat{d}_{n m, i}$ of $d_{n m, i}$ can be realized by substituting $y_{m}(k)$ from (2) in (14). The simplified solution for $\hat{d}_{n m, i}$ can be expressed as under,

$$
\hat{d}_{n m, i}=d_{n m, i}+\varepsilon_{n m, i},
$$

where $\varepsilon_{n m, i}$ represents the error in the estimate of $d_{n m, i}$. The estimation error $\varepsilon_{n m, i}$ contains contributions from additive noise $\left(\tilde{\eta}_{n m, i}\right)$, interference from superimposed information sequence of all the transmitters $\left(\tilde{b}_{n m, i}\right)$, and the interference from training sequence of cross channels $\left(\tilde{c}_{\tilde{\tilde{n}} m, i}\right)$. The estimation error is thus given by, $\varepsilon_{n m, i}=\tilde{c}_{\tilde{n} m, i}+\tilde{b}_{n m, i}+\tilde{\eta}_{n m, i}$; where,

$$
\begin{gathered}
\tilde{c}_{\tilde{n} m, i}=\frac{1}{T} \sum_{k=0}^{T-1}\left[\sum_{\substack{\tilde{n} \neq \\
\bar{n}}}^{N} \sum_{l=0}^{L-1} h_{\tilde{n} m}^{l} c_{\tilde{n}}(k-l)\right] \mathrm{e}^{-j \alpha_{i, \tilde{n}} k}, \\
\tilde{b}_{n m, i}=\frac{1}{T} \sum_{k=0}^{T-1}\left[\sum_{n=1}^{N} \sum_{l=0}^{L-1} h_{n m}^{l} b_{n}(k-l)\right] \mathrm{e}^{-j \alpha_{i, n} k}, \\
\tilde{\eta}_{n m, i}=\frac{1}{T} \sum_{k=0}^{T-1} \eta_{m}(k) \mathrm{e}^{-j \alpha_{i, n} k}
\end{gathered}
$$

Ignoring the inherent error $\varepsilon_{n m, i}$ in (19) leads to a non-realistic estimate of the channels. Moreover, the SI-LS estimation technique presented in the previous section is not optimized for sparse multipath channels. To address this issue, we propose a novel SI-MP algorithm. 
SI-MP Algorithm: Since the channel under consideration is sparse, a large number of taps in the channel vector $\mathbf{h}_{n m}$ is either zero or below the noise floor. Thus, a MP algorithm can be employed to estimate the sparse channel as proposed in [5]. Hence, to estimate the channels from the model in (15), the positions of non-zero taps are first determined and channel estimation is then carried out only for these specific non-zero positions. We describe the proposed SI-MP algorithm in the following paragraphs.

In (15), both $\mathbf{C}_{n}$ and $\mathbf{d}_{n m}$ are known, therefore, $\mathbf{d}_{n m}$ can be expanded as,

$$
\mathbf{d}_{n m}=\mathbf{c}_{n, o} h_{n m}^{0}+\mathbf{c}_{n, 1} h_{n m}^{1}+\mathbf{c}_{n, 2} h_{n m}^{2}+\ldots+\mathbf{c}_{n, \tilde{P}-1} h_{n m}^{\tilde{P}-1}
$$

where $\mathbf{c}_{n, i}$ is the $i^{\text {th }}$ column vector of $\mathbf{C}_{n}$. First, find the column in matrix $\mathbf{C}_{n}=\left[\mathbf{c}_{n, 0}, \mathbf{c}_{n, 1}, \ldots, \mathbf{c}_{n,\left(\tilde{P}_{-1}\right)}\right]$ that is best aligned with the output vector $\hat{\mathbf{d}}_{n m}$; let this vector be denoted by $\mathbf{c}_{q_{p}}$. Let $Q$ be the number of non-zero taps among a total of $L$ channel taps. The output vector $\hat{\mathbf{d}}_{n m}$ is spanned by total $Q$ columns of $\mathbf{C}_{n}$ that actually correspond to $Q$ non-zero entries of the sparse channel $\mathbf{h}_{n m}$. By projecting all columns of $\mathbf{C}_{n}$ on $\hat{\mathbf{d}}_{n m}$, we can find the best aligned column of $\mathbf{C}_{n}$ with $\hat{\mathbf{d}}_{n m}$ that will correspond to the position of one of the nonzero entries of $\mathbf{h}_{n m}$. In this way, we can find the location of non-zero entries present in the sparse channel $\mathbf{h}_{n m}$ in each iteration. Once the non-zero tap position of $\mathbf{h}_{n m}$ is determined, the value at that tap position can be found. The algorithm proceeds in the same way for each iteration. In order to find the non-zero tap positions of $\mathbf{h}_{n m}$ in the $q^{t h}$ iteration, the projection of $\mathbf{C}_{n}$ along $\hat{\mathbf{d}}_{n m}$ is found as,

$$
\gamma_{q}=\arg \max _{\substack{j=0, \ldots, \tilde{P}-1 \\ j \neq \gamma_{q-1}}} \frac{\left|\mathbf{c}_{n, j}^{*} \tilde{\mathbf{d}}_{n m, q-1}\right|^{2}}{\left\|\mathbf{c}_{n, j}\right\|_{\ell_{2}}^{2}},
$$

where $\|.\|_{\ell 2}$ represents the $\ell_{2}$ norm operation and $\mathbf{c}_{n, j}$ represents the $j^{\text {th }}$ column of $\mathbf{C}_{n}$. In the $q^{\text {th }}$ iteration, $\gamma_{q}$ represents the index of the best aligned column of $\mathbf{C}_{n}$ with $\hat{\mathbf{d}}_{n m}$ and corresponds to one of the non-zero taps of $\mathbf{h}_{n m}$. The projection of $\mathbf{C}_{n}$ in each iteration is computed along the residual error vector of previous iteration $\tilde{\mathbf{d}}_{n m, q-1}$. For the very first iteration, the residual error vector is $\tilde{\mathbf{d}}_{n m, o}=\hat{\mathbf{d}}_{\mathbf{n m}}$ and in the preceding iterations, its value is obtained as,

$$
\tilde{\mathbf{d}}_{n m, q}=\tilde{\mathbf{d}}_{n m, q-1}-\frac{\mathbf{c}_{n, \gamma_{q}}^{*} \tilde{\mathbf{d}}_{n m, q-1}}{\left\|\mathbf{c}_{n, \gamma_{q}}\right\|_{\ell_{2}}^{2}} \mathbf{c}_{n, \gamma_{q}} .
$$

The estimate of a non-zero tap of the channel $\tilde{h}_{n m}^{\gamma_{q}}$ at position $\gamma_{q}$ can thus be obtained as,

$$
\tilde{h}_{n m}^{\gamma_{q}}=\frac{\mathbf{c}_{\gamma_{q}}^{*} \tilde{\mathbf{d}}_{q-1}}{\left\|\mathbf{c}_{\gamma_{q}}\right\|_{\ell_{2}}^{2}} .
$$

The iterations continue until all the non-zero taps in $\tilde{\mathbf{h}}_{n m}$ are determined, or when the error residual in a specific iteration becomes less than agiven threshold, i.e., $\left\|\tilde{\mathbf{d}}_{q}\right\|<\epsilon$. In this algorithm, only non-zero taps of the sparse channel $\mathbf{h}_{n m}$ are estimated.

\section{LMMSE Equalizer for The Proposed Method}

The superimposed training sequence of each user terminal is known to the receiver, and this training sequence also gets convolved with the CIR along with the information sequence. Thus, before passing the signal to the equalizer's input, we need to remove the effect of the training sequence from the signal received at each antenna element of the receiver. TER performs this operation (as depicted in Fig. 1) in the following manner,

$$
\tilde{y}_{m}(k)=y_{m}(k)-\sum_{n=1}^{N} \sum_{l=0}^{L-1} \breve{h}_{n m}^{l} c_{n}(k-l),
$$

where $\breve{h}_{n m}^{l}$ represents the estimate of $l^{\text {th }}$ tap of channel from $n^{\text {th }}$ user terminal to $m^{\text {th }}$ antenna element of the receiver. The estimate of the channels can be obtained by using the techniques discussed in the previous sections, i.e., $\breve{\mathbf{h}}_{n m}=\hat{\mathbf{h}}_{n m}$ for the conventional SI-LS or $\breve{\mathbf{h}}_{n m}=\tilde{\mathbf{h}}_{n m}$ for the proposed SIMP. Once the effect of training sequence is removed, the signal is fed to the equalizer's input which estimates the information sequence of each user. For, the equalizers part at $m^{\text {th }}$ element of the receiver, the optimal equalizer's weights $\mathbf{w}_{m}$ can be obtained as in [30], and are given by,

$$
\mathbf{w}_{n m}=\left.\left(\breve{\mathbf{H}} \breve{\mathbf{H}}^{*}+2 \sigma_{n}^{2} \mathbf{I}\right)^{-1} \breve{\mathbf{H}}\right|_{(m-1)\left(L_{e}+L-1\right)+(\tau+1)},
$$

where $L_{e}$ denotes the length of equalizer, I denotes the $L_{e} \times L_{e}$ identity matrix, $\tau$ is the decision delay of the equalizer's mappers and $\left.\breve{\mathbf{H}}\right|_{i}$ is the $i^{t h}$ column of $\mathbf{H}$. The convolutional matrix $\breve{\mathbf{H}}$, having dimensions $n_{f} \times\left(L_{e}+L-1\right)$, is computed from the estimated channels' impulse response vectors as below,

$$
\breve{\mathbf{H}}_{n, m}=\left[\begin{array}{ccccccc}
\breve{h}_{n, m}^{0} & \breve{h}_{n, m}^{1} & \ldots & \breve{h}_{n, m}^{L-1} & 0 & \cdots & 0 \\
0 & \breve{h}_{n, m}^{0} & \breve{h}_{n, m}^{1} & \ldots & \breve{h}_{n, m}^{L-1} & \ddots & \vdots \\
\vdots & \ddots & \ddots & \ddots & \ddots & \ddots & 0 \\
0 & \ldots & 0 & \breve{h}_{n, m}^{0} & \breve{h}_{n, m}^{1} & \cdots & \breve{h}_{n, m}^{L-1}
\end{array}\right] .
$$

Subsequently, the composite convolutional matrix $\breve{\mathbf{H}}$ of the MIMO system is given as,

$$
\breve{\mathbf{H}}=\left[\begin{array}{cccc}
\breve{\mathbf{H}}_{1,1} & \breve{\mathbf{H}}_{1,2} & \cdots & \breve{\mathbf{H}}_{1, M} \\
\breve{\mathbf{H}}_{2,1} & \mathbf{H}_{2,2} & \cdots & \breve{\mathbf{H}}_{2, M} \\
\vdots & \vdots & \cdots & \vdots \\
\breve{\mathbf{H}}_{L, 1} & \breve{\mathbf{H}}_{L, 2} & \cdots & \breve{\mathbf{H}}_{L, M}
\end{array}\right] .
$$

Next, the estimate of transmitted information sequence from the $n^{\text {th }}$ transmitter can be obtained as,

$$
\tilde{\mathbf{b}}_{n}=\sum_{m=1}^{M} \sum_{i=0}^{L_{e}-1} w_{n m}^{i} \tilde{y}_{m}(k-i) .
$$

The output of the equalizer, $\tilde{\mathbf{b}}_{n}$, is then fed as an input to a decision mapper, as shown in Fig. 1, that performs mapping of the symbols according to the used modulation scheme with the decoded symbols represented by $\hat{\mathbf{b}}_{n}$. 


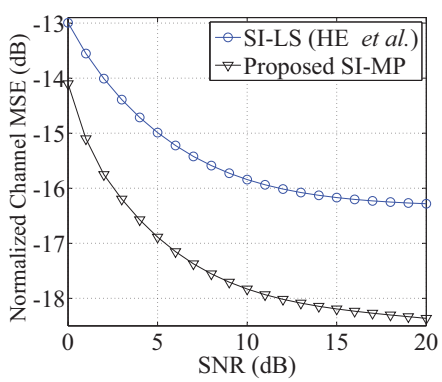

(a) MSE based comparison.

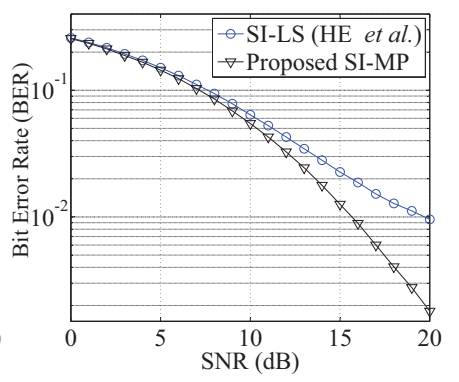

(b) BER based comparison.
Fig. 2: MSE and BER based comparative analysis between SILS and the proposed SI-MP, (Monte= 1000 runs, $K=900$, $L=14, Q=3, M=N=3, \sigma_{c}^{2} / \sigma_{b}^{2}=0.25, \tau=5$, and $\left.L_{e}=11\right)$.

\section{RESUlTS AND DiscusSiON}

In this section, a detailed numerical analysis of simulation results is presented by considering the normalized channel MSE (NCMSE) and BER as performance metrics. The NCMSE before Monte Carlo averaging is defined as,

$$
\mathrm{NCMSE}=\frac{\sum_{m=1}^{M} \sum_{n=1}^{N} \sum_{l=0}^{L-1}\left|\hat{h}_{n m}^{l}-h_{n m}^{l}\right|^{2}}{\sum_{m=1}^{M} \sum_{n=1}^{N} \sum_{l=0}^{L-1}\left|h_{n m}^{l}\right|^{2}},
$$

where $|$.$| represents the magnitude.$

We consider a $3 \times 3$ MIMO system with time invariant and frequency selective sub channels. The realization of all the channels $\mathbf{h}_{n m}$ is independently generated for each Monte Carlo run, by keeping a fixed level of sparsity, $Q / L$. The support (non-zero delay positions) of impulse response vector for all the channels is drawn independently from uniform distribution. The values of non-zero taps of a channel $h_{n m}$ follow a zero-mean Gaussian distribution. The number of resolvable multipath components is taken equal for all the channels, fixed at $L=14$ with variance $1 /(M(L+1))$. A periodic training sequence is generated following the $m$-sequence approach presented in [22]. A periodic base sequence with period $\tilde{P}=15$ is taken fixed as, $\{-1,-1,-1,1,1,1,1,-1,1,-1,1,1,-1,-1,1\}$, for all the simulation results. Zero mean white Gaussian noise is independently generated at each receiver satisfying a certain signal-to-noise (SNR) ratio. The SNR at $m^{\text {th }}$ receiver is defined as the ratio between variance of received signal $\sigma_{y_{m}}^{2}$ and variance of noise $\sigma_{\eta}^{2}$, i.e., $\mathrm{SNR}_{m}=\sigma_{y_{m}}^{2} / \sigma_{\eta}^{2}$. The information sequence of each transmitter is considered to be a zero-mean BPSK modulated signal i.e., $\mathbf{b}_{n} \in\{1,-1\}$. A MMSE equalizer (explained in previous section) is implemented to obtained estimate of information sequences at the receiver. The performance comparison, in terms of NCMSE and BER, of the first-order statistics based channel estimation technique (SI-LS) and the proposed channel estimation technique using matching pursuit algorithm (SI-MP) is shown in Fig. 2. It can be observed that the proposed technique outperforms SI-LS technique in terms of both NCMSE and BER. For the case of NCMSE, we have a performance gain of $2 \mathrm{~dB}$ at an SNR of 10

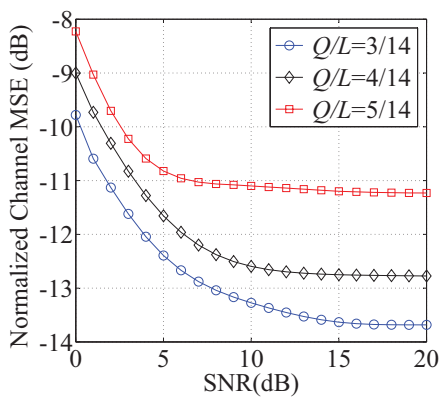

(a) Variation in sparsity.

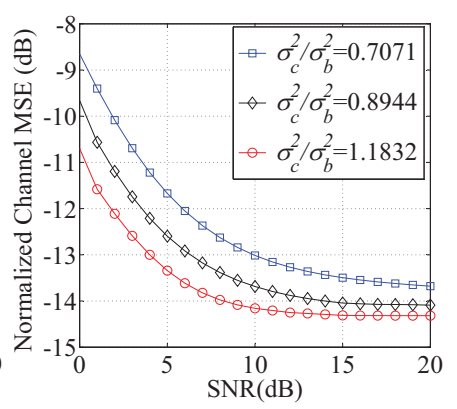

(b) Variation in TIR
Fig. 3: Effects of variation in TIR and channel sparsity on MSE performance of CE, (Monte $=1000$ runs, $K=900$, and $M=N=3)$.

$\mathrm{dB}$ for $Q / L=3 / 14$. For $\mathrm{BER}=10^{-2}$, the proposed technique gives a performance gain of about $3 \mathrm{~dB}$ as compared to SILS. This performance gain provided by the proposed technique arises due to the exploitation of the sparse nature of MIMO channels.

It can be depicted from Fig. 3 that the proposed technique performs better due to the use of prior knowledge about the sparse nature of the channel. To demonstrate the effect of variation in the channel's sparsity (i.e., $Q / L$ ), the MSE is plotted against SNR for different values of sparsity (i.e., $Q / L=2 / 14,3 / 14$, and 5/14) in Fig. 3(a) for the proposed SI-MP. For these plots, the training to information ratio (TIR) $\sigma_{c}^{2} / \sigma_{b}^{2}$ is set to unity. It can be observed that the performance of the proposed scheme increases as the channels become more and more sparse. To demonstrate the effect of variation in TIR $\left(\sigma_{c}^{2} / \sigma_{b}^{2}\right)$, the MSE versus SNR is plotted for different values of TIR by keeping the channel sparsity parameter $Q / L=4 / 14$ fixed, as shown in Fig. 3(b). It can be noted that by increasing the power of the training sequence, the MSE performance of the channel improves. However, decreasing the contribution of information sequence has an adverse effect on the BER performance of the system while decoding the information sequence, see Fig. 4. Therefore, the choice of an optimum value for TIR is required to satisfy a good tradeoff between MSE and BER performance.

\section{CONCLUSION}

A novel superimposed training sequence based estimation technique using a matching pursuit algorithm has been proposed for frequency-selective and time-invariant sparse MIMO communication channels. A detailed analysis based on the simulation results has been presented in terms of MSE and BER performance metrics. Furthermore, the effect of variation in the channels' sparsity level and the training to information power ratio has been investigated. It has been established that the proposed SI-MP technique outperforms the traditional SILS technique found in existing literature, for the case of sparse MIMO channels. It has been observed that the performance of the estimation technique is a function of degree of sparsity in 


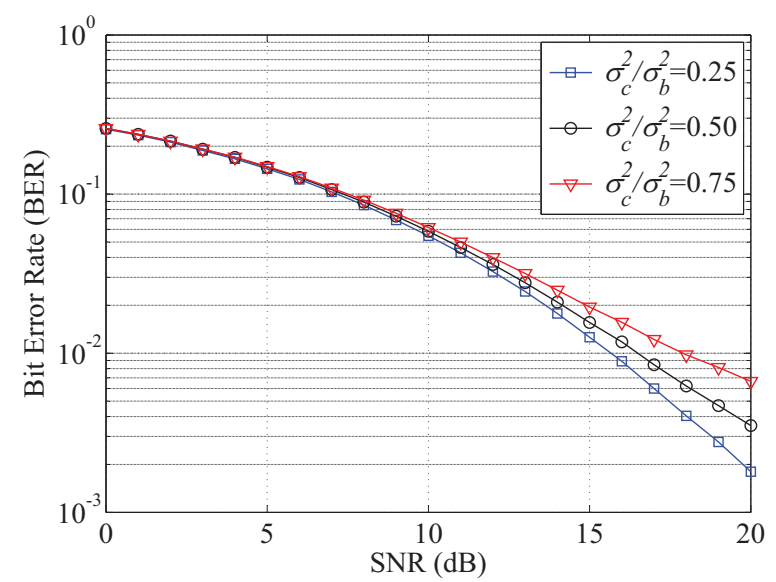

Fig. 4: Effect of variations in TIR on the BER performance, (Monte $=1000$ runs, $K=1500, Q / L=3 / 6, M=N=3$, $\tau=5$, and $L_{e}=8$ ).

the CIR. Hence can be improved with dynamic measure of the degree of sparsity at the receiver.

In our future work, we plan to extend this work for the estimation of time-variant sparse MIMO channels with SiT sequence.

\section{REFERENCES}

[1] E. Hossain, M. Rasti, H. Tabassum, and A. Abdelnasser, "Evolution toward 5G multi-tier cellular wireless networks: An interference management perspective," IEEE Wireless Comm., vol. 21, no. 3, pp. 118 127, Jun. 2014.

[2] Z. Gao, L. Dai, D. Mi, Z. Wang, M. A. Imran, and M. Z. Shakir "MmWave massive-MIMO-based wireless backhaul for the $5 \mathrm{G}$ ultradense network," IEEE Wireless Comm., vol. 22, no. 5, pp. 13-21, Oct. 2015.

[3] A. L. Swindlehurst, E. Ayanoglu, P. Heydari, and F. Capolino, "Millimeter-wave massive MIMO: the next wireless revolution?" IEEE Comm. Magazine, vol. 52, no. 9, pp. 56-62, Sep. 2014.

[4] A. Alkhateeb, O. E. Ayach, G. Leus, and R. W. Heath, "Channel estimation and hybrid precoding for millimeter wave cellular systems," IEEE J. of Selected Topics in Signal Processing, vol. 8, no. 5, pp. 831846, Oct. 2014.

[5] S. F. Cotter and B. D. Rao, "Sparse channel estimation via matching pursuit with application to equalization," IEEE Trans. on Comm., vol. 50, no. 3, pp. 374-377, Mar. 2002.

[6] S. J. Nawaz, K. I. Ahmed, M. N. Patwary, and N. M. Khan, "Superimposed training-based compressed sensing of sparse multipath channels,' IET Comm., vol. 6, no. 18, pp. 3150-3156, Dec. 2012

[7] F. K. Lee and P. J. McLane, "Design of nonuniformly spaced tappeddelay-line equalizers for sparse multipath channels," Comm., IEEE Trans. on, vol. 52, no. 4, pp. 530-535, 2004.

[8] J. Ying, J. Zhong, M. Zhao, and Y. Cai, "Turbo equalization based on compressive sensing channel estimation in wideband HF systems," in Proc. of Int. Conf. on Wireless Comm. Signal Processing, Oct. 2013, pp. $1-5$.

[9] M. Kocic, D. Brady, and M. Stojanovic, "Sparse equalization for realtime digital underwater acoustic communications," in Proc. of OCEANS Conf., 1995.

[10] J. Zhao, W. Meng, and S. Jia, "Sparse underwater acoustic OFDM channel estimation based on superimposed training," J. of Marine Science and Application, vol. 8, no. 1, pp. 65-70, 2009.

[11] N. Seshadri, "Joint data and channel estimation using blind Trellis search techniques," IEEE Trans. on Comm., vol. 42, no. 234, pp. 1000-1011, Feb. 1994.

[12] S. Talwar, M. Viberg, and A. Paulraj, "Blind estimation of multiple cochannel digital signals using an antenna array," IEEE Signal Processing Letters, vol. 1, no. 2, pp. 29-31, Feb. 1994.
[13] J. K. Tugnait, "Blind estimation of digital communication channel impulse response," IEEE Trans. on Comm., vol. 42, no. 234, pp. 16061616, Feb. 1994

[14] E. Aktas and U. Mitra, "Semiblind channel estimation for CDMA systems with parallel data and pilot signals," IEEE Trans. on Comm., vol. 52, no. 7, pp. 1102-1112, Jul. 2004

[15] W. Hou and B. Champagne, "Semiblind channel estimation for OFDM/OQAM systems," IEEE Signal Processing Letters, vol. 22, no. 4 pp. 400-403, Apr. 2015.

[16] T. Cui and C. Tellambura, "Semiblind channel estimation and data detection for OFDM systems with optimal pilot design," IEEE Trans. on Comm., vol. 55, no. 5, pp. 1053-1062, May 2007.

[17] E. Bjornson and B. Ottersten, "A framework for training-based estimation in arbitrarily correlated Rician MIMO channels with Rician disturbance," IEEE Trans. on Signal Processing, vol. 58, no. 3, pp. 1807-1820, Mar. 2010

[18] C. Fragouli, N. Al-Dhahir, and W. Turin, "Training-based channel estimation for multiple-antenna broadband transmissions," IEEE Trans. on Wireless Comm., vol. 2, no. 2, pp. 384-391, Mar. 2003.

[19] C. Murthy, A. Jagannatham, and B. Rao, "Training-based and semiblind channel estimation for MIMO systems with maximum ratio transmission," IEEE Trans. on Signal Processing, vol. 54, no. 7, pp. 2546-2558, Jul. 2006.

[20] X. Xie, M. Peng, F. Gao, and W. Wang, "Superimposed training based channel estimation for uplink multiple access relay networks," IEEE Trans. on Wireless Comm., vol. 14, no. 8, pp. 4439-4453, Aug. 2015.

[21] B. Farhang-Boroujeny, "Pilot-based channel identification: proposal for semi-blind identification of communication channels," IET Electronics Letters, vol. 31, no. 13, pp. 1044-1046, 1995.

[22] S. He, J. K. Tugnait, and X. Meng, "On superimposed training for MIMO channel estimation and symbol detection," IEEE Trans. on Signal Processing, vol. 55, no. 6, pp. 3007-3021, 2007.

[23] E. J. Candes and T. Tao, "Decoding by linear programming," IEEE Trans. on Information Theory, vol. 51, no. 12, pp. 4203-4215, Dec. 2005.

[24] E. J. Candes, J. Romberg, and T. Tao, "Robust uncertainty principles: exact signal reconstruction from highly incomplete frequency information," IEEE Trans. on Information Theory, vol. 52, no. 2, pp. 489-509, Feb. 2006.

[25] D. L. Donoho, "Compressed sensing," IEEE Trans. on Information Theory, vol. 52, no. 4, pp. 1289-1306, Apr. 2006.

[26] W. U. Bajwa, J. Haupt, A. M. Sayeed, and R. Nowak, "Compressed channel sensing: A new approach to estimating sparse multipath channels," Proc. of the IEEE, vol. 98, no. 6, pp. 1058-1076, Jun. 2010.

[27] G. Taubock and F. Hlawatsch, "A compressed sensing technique for ofdm channel estimation in mobile environments: Exploiting channel sparsity for reducing pilots," in Proc. of IEEE Int. Conf. on Acoustics, Speech, and Signal Processing., Mar. 2008, pp. 2885-2888.

[28] C. R. Berger, Z. Shengli, J. C. Preisig, and P. Willett, "Sparse channel estimation for multicarrier underwater acoustic communication: From subspace methods to compressed sensing," IEEE Trans. on Signal Processing, vol. 58, no. 3, pp. 1708-1721, Mar. 2010.

[29] J. K. Tugnait and W. Luo, "On channel estimation using superimposed training and first-order statistics," in Proc. of IEEE Int. Conf. on Acoustics, Speech, and Signal Processing, vol. 4, 2003, pp. IV-624.

[30] S. Chen, A. Livingstone, and L. Hanzo, "Minimum bit-error rate design for space-time equalization-based multiuser detection," IEEE Trans. on Comm., vol. 54, no. 5, pp. 824-832, 2006 